\title{
Optimal schedule of bacillus calmette-guerin for non-muscle-invasive bladder cancer: a meta-analysis of comparative studies
}

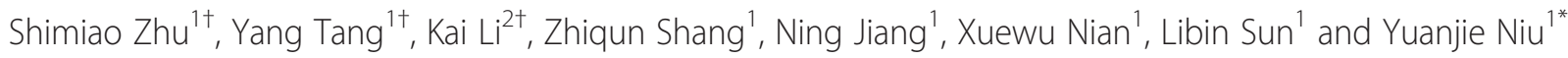

\begin{abstract}
Background: To explore the necessity of maintenance, efficacy of low-dose and superiority of various combination therapies of Bacillus Calmette-Guérin (BCG) in treatment of superficial bladder cancer (BCa).

Methods: Comprehensive searches of electronic databases (PubMed, Embase, and the Cochrane Library) were performed, then a systematic review and cumulative meta-analysis of 21 randomized controlled trials (RCTs) and 9 retrospective comparative studies were carried out according to predefined inclusion criteria.

Results: Significantly better recurrence-free survivals (RFS) were observed respectively in patients who received BCG maintenance, standard-dose and BCG plus epirubicin therapy comparing to those received induction, low-dose and BCG alone. BCG maintenance therapy was also associated with significantly better progression-free survival (PFS), but there were more incidences of adverse events. Pooled results showed no remarkable advantage of BCG combined with Mitomycin C or with interferon $a-2 b$ in improving oncologic outcomes. Sensitivity-analyses stratified by study-design and tumor stage led to very similar overall results and often to a decrease of the between-study heterogeneity. Our data confirmed that non-RCT only affected strength rather than direction of the overall results.

Conclusions: All patients with superficial BCa should be encouraged to accept BCG maintenance therapy with standard-dose if well tolerated. Patients can benefit from BCG combined with epirubicin but not from BCG combined with Mitomycin C or interferon a-2b.
\end{abstract}

Keywords: Bacillus Calmette-Guérin, Non-muscle-invasive bladder cancer, Maintenance therapy, Low-dose, Combination therapy, Prognosis

\section{Background}

More than 30 years ago, intravesical Bacillus CalmetteGuérin (BCG) was first proposed by Morales [1]. Since then, BCG therapy has been demonstrated to be the most effective treatment in the prevention of the recurrence and progression of superficial bladder cancer (BCa), especially for high-risk non-muscle-invasive bladder cancer (NMIBC) [2]. Despite its well-recognized efficacy, many questions remain suspended and among them, the following ones should be noted: 1) the necessity of maintenance BCG therapy; 2) the efficacy of low-

\footnotetext{
* Correspondence: yuanjieniu68@hotmail.com

${ }^{\dagger}$ Equal contributors

'Department of Urology, Tianjin Institute of Urology, 2nd Hospital of Tianjin Medical University, Pingjiang Road 23, Tianjin, People's Republic of China Full list of author information is available at the end of the article
}

dose BCG; and 3) the superiority of combination therapy of BCG.

Previous studies showed that only maintenance BCG could benefit patients in reducing tumor progression $[3,4]$. However, the results were from studies comparing maintenance BCG with Mitomycin C (MMC) other than BCG induction, and high-level direct evidence supporting maintenance therapy was still absent. The maintenance BCG has been compromised because of serious side-effects (e.g., BCG sepsis and BCG-induced cystitis). Then, low-dose BCG [5-7], recognized to be accompanying with reduced side effects, was introduced. Higher recurrence and progression rates were observed in lowdose group comparing with standard-dose group [5-7], nevertheless, the wide confidential intervals $(\mathrm{CI})$ of individual studies made us failed to detect significant

\section{() Biomed Central}


difference between the two groups. So, it's necessary for us to perform meta-analyses to systematically evaluate the necessity of maintenance BCG and the efficacy of low-dose BCG therapy.

Additionally to optimize the BCG therapy schedules, efficacy of BCG combination therapies (e.g. BCG plus MMC, BCG plus epirubicin and BCG plus interferon $\alpha-2 b(\operatorname{IFN}-\alpha 2 b))$ were also evaluated in this metaanalysis. Many studies had been addressed to improve BCG efficacy by combining with other remedies [8], however, no consistent conclusion was obtained. Thus, the systematic syntheses were addressed to explore the optimal schedule and dose of BCG prescription for NMIBC.

\section{Methods}

\section{Search strategy and study selection}

This meta-analysis was conducted according to the Preferred Reporting Items for Systematic Reviews and MetaAnalyses (PRISMA) [9]. A systematic search of Medline, Embase, and the Cochrane Library was performed using all possible combinations of the following keywords: 1) 'Low-Dose' or 'Low doses' or 'maintenance instillation' or 'maintenance' or 'Mitomycin C' or 'MMC' or 'Epirubicin' or 'interferon' or 'IFN' or 'combination therapy' and 'BCG' or 'Bacillus Calmette-Guérin' and 'bladder cancer' or 'Transitional cell carcinoma of bladder' or 'urothelial carcinoma of bladder'; 2) and 'BCG' or 'Bacillus Calmette-Gu érin' and 'bladder cancer' or 'Transitional cell carcinoma of bladder' or 'urothelial carcinoma of bladder'. To perform an extensive search, no language, publication year, or other limits were used. The last quest was updated on March 7, 2013. Reference lists of relevant reviews were hand-searched to identify additional studies.

Eligible studies had to meet the following inclusion criteria: (1) The diagnosis of $\mathrm{BCa}$ had to be confirmed pathologically; (2) All patients should be confirmed as NMIBC; (3) Included studies had to provide comparative data; and (4) Only the most recent trials with the greatest number of patients was chosen when overlapped subjects were selected in more than one study.

\section{Data extraction and quality assessment}

Informations were carefully extracted from all eligible publications independently by two authors, and all disagreements were resolved by the third reviewer (Niu) until consensus was achieved on all items. The following data were considered in eligible studies: author name, year and country of the trials, numbers of case and control subjects, age, duration of follow-up, Treatment schedules and doses of medicines, Hazard Ratios (HRs) or Risk Ratios (RRs) and corresponding 95\% CIs of each comparisons.
The methodological quality of RCT was assessed by the Jadad scale [10], considering that a high quality RCT should get more than 3 points. The retrospective studies were assessed by the Newcastle-Ottawa scale [11]. Observational studies achieving six or more stars were considered to be of high quality. Assessments were addressed independently by two authors and the disagreements between authors are resolved by discussion. Additionally, all included studies were also evaluated according to the level of evidence (LOE) stated by Phillips et al. [12].

\section{Statistical analysis}

The recurrence-free survival (RFS) and Progression-free survival (PFS) were evaluated to assess the effects of various treatment schedules and doses. The HRs or Risk Differences (RDs) were used to compare all dichotomous variables. HRs were estimated by different methods depending on the data provided in the publication. The simplest method was to collect reported HRs and their 95\% CIs in texts. If those data were not available, we looked for the total number of events, the number of patients at risk in each group and the logrank statistic or its $\mathrm{P}$ value allowing calculation of an approximation of the HR estimate. If data were only available in the form of survival curve (SC), we extracted from the survival rates at some specified times so as to estimate the HR value and its variance, assuming that the rate of patients censored was constant during the study follow-up [13]. If the data mentioned above were not available, risk ratio should be considered as the last option. To avoid potential publication bias caused by cherry-picking, comparative studies were identified no matter of study design. Then, sensitivity analysis stratified by study-design was conducted to decrease potentially introduced bias by observational studies. In addition, sensitivity analysis stratified by tumor stage was addressed to narrow the population that was suitable for indicated treatment.

Statistical heterogeneity between trials included in the meta-analysis was assessed using Cochrane's Q statistic [14] . And the inconsistency was quantified by $I^{2}$ statistic $(100 \% \times[(\mathrm{Q}-\mathrm{df}) / \mathrm{Q}])$, higher value denoting greater degree of heterogeneity [15]. Fixed-effects model was used when heterogeneity was not observed; otherwise, randomeffects model was used. Fixed-effects model, using the Mantel-Haenszel method, assumed that studies were sampled from populations with the same effect size; whereas the random-effects model, using the DerSimonian and Laird method, considered that studies were taken from populations with different effect sizes.

Publication bias was evaluated using Begg adjusted rank correlation test and Egger linear regression test. All statistical analyses were conducted using STATA (version 11.0; College Station, Texas) and Review Manage (version 5.1; 
The Cochrane Collaboration, Oxford). Two-tailed P value of less than .05 was considered statistically significant.

\section{Results}

\section{Literature search and characteristics of the included} studies

After removing 692 duplicates, we screened 755 potentially relevant articles. The final number of papers included in the meta-analysis was 30, list of studies excluded and reasons were shown in Figure 1. Of these, nine were identified to explore the necessity of BCG maintenance [16-24], seven investigate the efficacy of low-dose BCG [5-7,25-28], and the remainders address the effects of BCG combination therapy [29-42]. There were 21 randomized-controlled trails (RCTs), eight retrospective studies and one case-series study. Search of the references listed in reviews did not yield any further available studies. Agreement between the two reviewers was $96 \%$ for study selection and $96 \%$ for quality assessment of trials.

The characteristics of included studies were shown in Tables 1, 2 and 3. Among the RCTs, there were 5 high quality studies (level of evidence: 2b) [6,22,24,33,38]. The severe side-effects that can be easily recognized by both patients and researchers made most of studies failed to use double-blind method, which should be responsible for the low-quality of included RCTs. Only 1

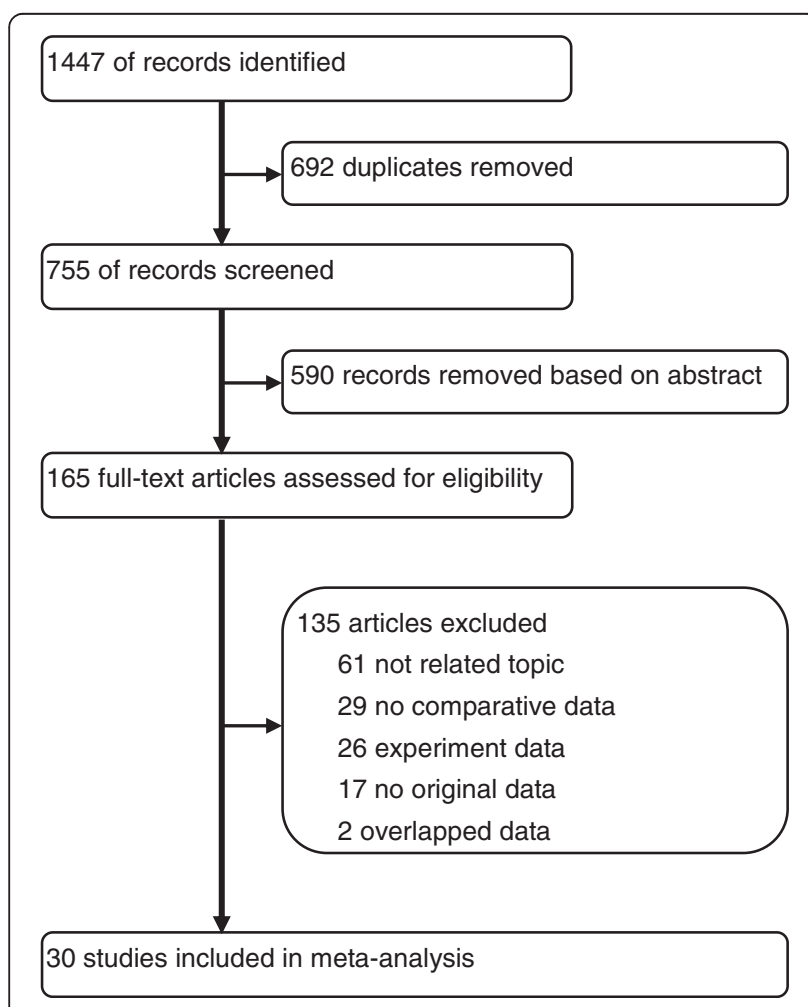

Figure 1 Flowchart of selecting process for meta-analysis. retrospective studies which didn't adopt appropriate protocol for treatment assignment and used historical series as controls were recognized as low quality caseseries study (level of evidence: 4) [42]. The results of quality assessment were listed in Additional file 1: Table S2 and Table S3.

\section{Meta-analysis results \\ BCG maintenance vs. induction alone}

In this meta-analysis of comparative studies, nine studies were identified to investigate the necessity of BCG maintenance therapy [16-24]. Pooled results of data that assessed tumor recurrence rate showed that BCG maintenance therapy could significantly improve RFS (HR $=0.516$; 95\% CI 0.425-0.627; $P<0.0001$ ) (Figure $2 \mathrm{~A}$ ), comparing with BCG induction. Cancer progression rates were investigated in 7 studies, and a notable advantage of BCG maintenance in improving PFS was also observed $(\mathrm{HR}=0.740$; 95\% CI 0.572-0.957; $P=0.022$ ) (Figure 2B). Five studies were available to explore the RFS and PFS in high-risk NMIBC patients underwent BCG maintenance or induction therapy $[16,18,19,21,22]$, significant differences were presented between the two treatment schedules (RFS: $\mathrm{HR}=0.515$, 95\% CI 0.411-0.646, $P<0.0001$; PFS: $\mathrm{HR}=0.722$, 95\% CI 0.548-0.951, $P=0.020$ ) (Table 4). No heterogeneity among included studies was significant for the above all analyses (Table 4).

\section{Low-dose vs. standard-dose BCG}

In term of RFS, six studies [5-7,25-28] including 998 cases and 1018 controls were identified to comparing low dose BCG to standard doses, cumulated result showed a remarkable difference opposing to low-dose BCG (HR=1.162; 95\% CI 1.051-1.285; $P=0.003$ ) (Figure 3A). Pooling data of 5 studies [5-7,25,26,28] including 1,942 patients that reported tumor progression rate showed the PFS was lower in low-dose BCG than in control group, but this difference was not statistically significant (HR=1.151; 95\% CI 0.853-1.554; $P=0.356$ ) (Figure 3B). Significant heterogeneity was detected in neither analysis above (Table 4).

\section{$B C G$ plus mitomycin $C$ vs. BCG alone}

Six studies involving 979 patients with superficial BCa were identified to investigate the difference between therapy schedules of BCG alone and BCG plus MMC [29-34], synthesized data showed no significantly better RFS in patients accepted combined therapy ( $\mathrm{HR}=0.726$; 95\% CI 0.490-1.075; $P=0.110$ ) (Figure 4A). High-risk NMIBC was evaluated in 3 of above 5 studies [30,33,34], also no significant result was observed ( $\mathrm{HR}=0.852$; $95 \%$ CI $0.449-1.617 ; P=0.624)$. Tumor progression rate was available in 3 studies, in these studies, only high-risk NMIBC patients were included [30,33,34]. Pooled analysis 
Table 1 Characteristics of studies investigating the effects of maintenance of BCG

\begin{tabular}{|c|c|c|c|c|c|c|c|}
\hline First author, yr & $\begin{array}{l}\text { Design, } \\
\text { LOE }\end{array}$ & $\begin{array}{c}\text { No. of } \\
\text { Case/Control }\end{array}$ & $\begin{array}{c}\text { Age } \\
\text { Case/Control* }\end{array}$ & Disease category & $\begin{array}{l}\text { Follow-up, mo } \\
\text { Case/Control* }\end{array}$ & Strain & $\begin{array}{l}\text { Treatment dose }(\mathrm{mg}) \\
\text { Maintenance/Induction }\end{array}$ \\
\hline Badalament 1987 [23] & $\mathrm{RCT}, 1 \mathrm{~b}$ & $47 / 46$ & 62.0/63.5 & Superficial BCa & $22(3-44)$ & Pasteur & $120 / 120$ \\
\hline Koga 2010 [18] & $\mathrm{RCT}, 1 \mathrm{~b}$ & $24 / 27$ & NA & High-risk NMIBC & $26.5 / 28.7$ & Tokyo & $80 / 80$ \\
\hline Hinotsu 2011 [16] & $\mathrm{RCT}, 1 \mathrm{~b}$ & $41 / 42$ & NA & Recurrent or multiple NMIBC & 24 & Connaught & $80 / 40$ \\
\hline Lamm 2000 [22] & $\mathrm{RCT}, 1 \mathrm{~b}$ & 192/192 & $66.8 / 67.0$ & Recurrent $\mathrm{Ta}, \mathrm{T} 1$ and $\mathrm{CIS}$ & $119.1 / 120.3$ & Connaught & $80 / 40$ \\
\hline Palou 2001 [21] & $\mathrm{RCT}, 1 \mathrm{~b}$ & $65 / 61$ & 65.0/63.0 & $\mathrm{CIS}$ and/or high grade $\mathrm{BCa}$ & $77.8(7-120)$ & Connaught & $81 / 81$ \\
\hline Hudson 1987 [24] & $\mathrm{RCT}, 1 \mathrm{~b}$ & $21 / 21$ & $60.0 / 62.0$ & Superficial BCa & $16.1 \pm 1.4$ & Pasteur & $120 / 120$ \\
\hline Andius 2004 [20] & $R, 2 b$ & $52 / 80$ & $74(31-96)$ & NMIBC without CIS & $44(4-155)$ & Danish/OncoTice & NA \\
\hline Okamura 2011 [17] & $R, 2 b$ & $48 / 27$ & $64.0 / 68.0$ & NMIBC without CIS & $102 / 66$ & Tokyo 172 & $80 / 80$ \\
\hline Decobert 2008 [19] & $R, 2 b$ & $31 / 40$ & $64.3 / 69.5$ & High-risk NMIBC & 31 & Pacis & $120 / 120$ \\
\hline
\end{tabular}

LOE Level of evidence, $R C T$ Randomized-controlled trial, $R$ Retrospective, NMIBC Non-muscle-invasive bladder cancer, BCa Bladder cancer, CIS Carcinoma in suit, NA Not available, Case/Control، BCG maintenance/Induction.

* Mean or median. 
Table 2 Characteristics of studies investigating the effects of low-dose BCG

\begin{tabular}{|c|c|c|c|c|c|c|c|c|}
\hline First author, yr & $\begin{array}{l}\text { Design, } \\
\text { LOE }\end{array}$ & $\begin{array}{c}\text { No. of } \\
\text { Case/Control }\end{array}$ & $\begin{array}{c}\text { Age } \\
\text { Case/Control* }\end{array}$ & Disease category & $\begin{array}{l}\text { Follow-up, mo } \\
\text { Case/Control* }\end{array}$ & Strain & $\begin{array}{l}\text { Treatment dose }(\mathrm{mg}) \\
\text { Case/Control }\end{array}$ & $\begin{array}{c}\text { Maintenance }^{* *} \\
\text { Case/Control }\end{array}$ \\
\hline Ojea 2007 [25] & $\mathrm{RCT}, 1 \mathrm{~b}$ & $139 / 142$ & $64.9 / 65.1$ & Intermediate-Risk NMIBC & $61.2 / 57.3$ & Connaught & $13.5 / 27$ & Yes/Yes \\
\hline Yalcinkaya 1998 [7] & $\mathrm{RCT}, 1 \mathrm{~b}$ & $25 / 25$ & $56.3 / 55.3$ & Superficial BCa & $26.8 / 31.6$ & Connaught & $54 / 81$ & No/No \\
\hline Kumar 2002 [6] & $\mathrm{RCT}, 2 \mathrm{~b}$ & $13 / 13$ & $55.9 / 56.7$ & NMIBC without CIS & $24(12-30)$ & Danish 1331 & $40 / 120$ & No/No \\
\hline Oddens 2013 [28] & $\mathrm{RCT}, 1 \mathrm{~b}$ & $678 / 677$ & $68.0 / 67.0$ & Intermediate and high risk NMIBC & 85.2 & OncoTice & $27 / 81$ & Yes/Yes \\
\hline Takashi 1995 [27] & $R, 2 b$ & $37 / 37$ & $68.1 / 61.4$ & Superficial BCa & 32 & Tokyo 172 & $40 / 80$ & $\mathrm{No} / \mathrm{No}$ \\
\hline Yoneyama 2008 [5] & $R, 2 b$ & $65 / 85$ & $68.3 / 65.5$ & NMIBC without CIS & $42.2 / 90.7$ & Tokyo 172 & $40 / 80$ & $\mathrm{No} / \mathrm{No}$ \\
\hline Irie 2003 [26] & $R, 2 b$ & $41 / 39$ & $61.6 / 62.2$ & Superficial BCa & $27.5 / 20.0$ & Tokyo 172 & $40 / 80$ & $\mathrm{No} / \mathrm{No}$ \\
\hline
\end{tabular}

LOE Level of evidence, RCT Randomized-controlled trial, $R$ Retrospective, NMIBC Non-muscle-invasive bladder cancer, BCa Bladder cancer, Case/Control, Low-dose/Standard-dose BCG.

* Mean or median.

** Maintenance is defined as any instillation beyond the induction course. 


\begin{tabular}{|c|c|c|c|c|c|c|c|c|}
\hline \multirow[t]{2}{*}{ First author, yr } & \multirow{2}{*}{$\begin{array}{l}\text { Design, } \\
\text { LOE }\end{array}$} & \multirow{2}{*}{$\begin{array}{c}\text { No. of } \\
\text { Case/Control }\end{array}$} & \multirow{2}{*}{$\begin{array}{c}\text { Age }^{*} \\
\text { Case/Control }\end{array}$} & \multirow[t]{2}{*}{ Disease category } & \multirow{2}{*}{$\begin{array}{l}\text { Follow-up, mo } \\
\text { Case/Control* }\end{array}$} & \multirow[t]{2}{*}{ Strain } & \multicolumn{2}{|c|}{ Treatment schedules } \\
\hline & & & & & & & Case & Control \\
\hline \multicolumn{9}{|c|}{ BCG plus mitomycin C vs. BCG alone (Case vs. Control) } \\
\hline Kaasinen 2003 [34] & $\mathrm{RCT}, 1 \mathrm{~b}$ & $159 / 145$ & $71.0 / 69.9$ & $\mathrm{CIS}$ & $56.3(1.9-97.3)$ & Connaught & $\begin{array}{c}6 \text { weekly BCG plus alternating BCG } \\
\text { and MMC monthly }\end{array}$ & $\begin{array}{l}6 \text { weekly BCG plus } \\
\text { monthly BCG }\end{array}$ \\
\hline Stasi 2006 [33] & $\mathrm{RCT}, 1 \mathrm{~b}$ & $107 / 105$ & $66.0 / 67.0$ & High-risk NMIBC & $91 / 84$ & Connaught & $\begin{array}{c}2 \text { weekly BCG and } 1 \text { week MMC as } \\
\text { one cycle for three cycles** }\end{array}$ & 6 weekly BCG** \\
\hline Oosterlinck 2011 [30] & $\mathrm{RCT}, 1 \mathrm{~b}$ & $48 / 48$ & $68.0 / 70.0$ & $\mathrm{CIS}$ & 56.4 & Tice & 6 weekly MMC plus 6 weekly BCG** & $\begin{array}{l}6 \text { weekly BCG plus } 3 \\
\text { weekly rest and BCG** }\end{array}$ \\
\hline Gulpinar 2012 [29] & $\mathrm{RCT}, 1 \mathrm{~b}$ & $25 / 26$ & $58.2 / 58.0$ & Intermediate-Risk NMIBC & $41.3 / 40.9$ & Tice & $\begin{array}{l}\text { Perioperative MMC plus } 6 \text { weekly } \\
\text { BCG }\end{array}$ & 6 weekly BCG \\
\hline El Mohsen 2010 [32] & $\mathrm{RCT} 1 \mathrm{~b}$ & $29 / 27$ & $47.5 / 48.1$ & Superficial BCa & 30 & NA & $\begin{array}{l}\text { Perioperative MMC plus } 4 \text { weekly } \\
\text { MMC plus monthly BCG for } 1 \text { year }\end{array}$ & $\begin{array}{l}6 \text { weekly BCG plus } \\
\text { monthly BCG for } 1 \text { year }\end{array}$ \\
\hline Badalato 2011 [31] & $R, 2 b$ & $48 / 212$ & $69.6 / 69.6$ & Superficial BCa & $33.0 / 43.6$ & Connaught & $\begin{array}{l}\text { Perioperative MMC plus } 6 \text { weekly } \\
\qquad \text { BCG** }^{* *}\end{array}$ & 6 weekly BCG ** \\
\hline \multicolumn{9}{|c|}{ BCG plus mitomycin C vs. mitomycin C alone (Case vs. Control) } \\
\hline Rintala 1996 [37] & $\mathrm{RCT}, 1 \mathrm{~b}$ & $95 / 93$ & $68 / 69$ & Rapidly recurring NMIBC & $34(1-76)$ & Pasteur & $\begin{array}{c}\text { Alternating courses of } \mathrm{MMC} \text { and } \mathrm{BCG} \\
\text { monthly during year } 1 \text { and every } 3 \\
\text { months during year } 2\end{array}$ & $\begin{array}{l}\text { MMC monthly during } \\
\text { year } 1 \text { and every } 3 \\
\text { months during year } 2\end{array}$ \\
\hline Jarvinen 2012 [35] & $\mathrm{RCT}, 1 \mathrm{~b}$ & $28 / 40$ & $66 / 68$ & $\mathrm{CIS}$ & 408 & Pasteur & Ditto & Ditto \\
\hline Witjes 1998 [36] & $\mathrm{RCT}, 1 \mathrm{~b}$ & $90 / 92$ & NA & Intermediate-Risk NMIBC & $32(2-65)$ & Tice & $\begin{array}{l}4 \text { weekly MMC followed by } 6 \text { weekly } \\
\text { BCG }\end{array}$ & 10 weekly MMC \\
\hline \multicolumn{9}{|c|}{ BCG plus Epirubicin vs. BCG alone (Case vs. Control) } \\
\hline Cai 2008 [38] & $\mathrm{RCT}, 1 \mathrm{~b}$ & $80 / 81$ & $73.9 / 69.8$ & High-risk NMIBC & $15.3 / 14.8$ & OncoTice & $\begin{array}{l}\text { Perioperative epirubicin plus delayed } \\
\text { BCG }\end{array}$ & $\begin{array}{c}6 \text { weekly BCG and every } \\
3 \text { months for } \\
\text { maintenance }\end{array}$ \\
\hline Bilen 2000 [40] & $\mathrm{RCT}, 1 \mathrm{~b}$ & $20 / 21$ & $57 / 53$ & High-risk NMIBC & 18(9-24) & Connaught & $\begin{array}{c}\text { Sequential instillation of BCG and } \\
\text { epirubicin for } 8 \text { weeks }\end{array}$ & 6 weekly BCG \\
\hline Tozawa 2001 [39] & $R, 2 b$ & $24 / 50$ & $67.9 / 65.6$ & Superficial BCa without CIS & $6-36$ & Tokyo & $\begin{array}{l}\text { Epirubicin and BCG were instilled by } \\
\text { turns once a week for } 12 \text { weeks }\end{array}$ & $\begin{array}{l}6 \text { weekly plus six } \\
\text { monthly BCG }\end{array}$ \\
\hline \multicolumn{9}{|c|}{ BCG plus Interferon $a-2 b$ vs. BCG alone (Case vs. Control) } \\
\hline Nepple 2010 [41] & $\mathrm{RCT}, 1 \mathrm{~b}$ & $346 / 324$ & 68.4 & Superficial BCa & NA & Tice & 6 weekly BCG plus IFN** & 6 weekly BCG ** \\
\hline Bazarbashi 2000 [42] & $C-S, 4$ & $37 / 18$ & $59 / 58$ & Superficial BCa & $26.2 / 23.8$ & Connaught & $\begin{array}{c}\text { Weekly sequential BCG and IFN for } 8 \\
\text { weeks }\end{array}$ & 6 weekly BCG \\
\hline
\end{tabular}

LOE Level of evidence, RCT Randomized-controlled trial, $R$ Retrospective, C-S Case-series, NMIBC Non-muscle-invasive bladder cancer, BCa Bladder cancer, CIS Carcinoma in suit, NA Not available. * Mean or median.

** Patients were rendered bladder cancer-free were given maintenance courses, Maintenance is defined as any instillation beyond the induction course. 

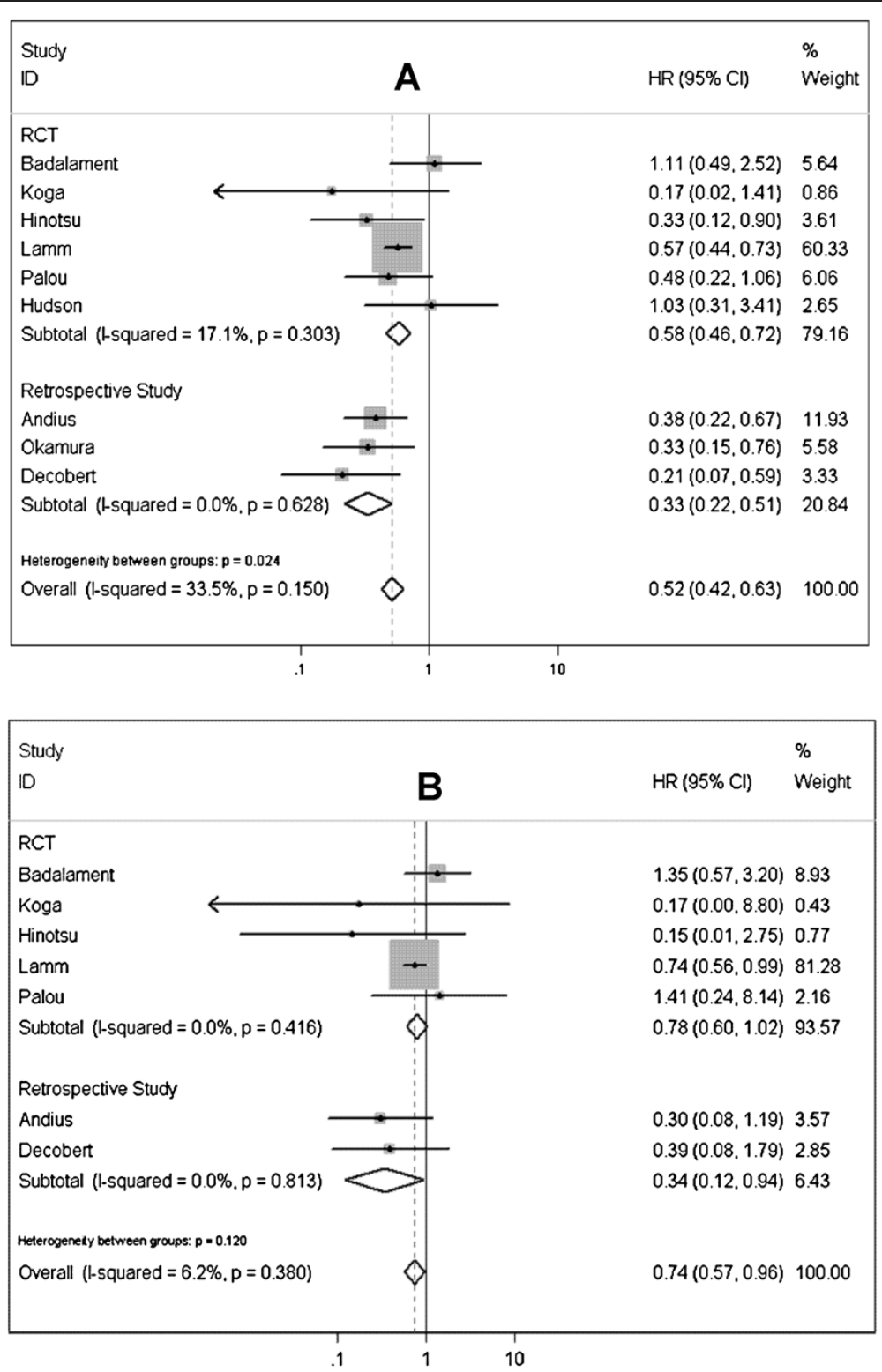

Figure 2 Forest plots of pooled results (A, recurrence-free survival; B, progression-free survival) for the maintenance group and non-maintenance group.

didn't show significantly different outcome between BCG plus Mitomycin C and BCG alone (HR=0.602; 95\% CI 0.181-1.999; $P=0.407$ ) (Figure 4B). There were significant heterogeneities in all above 3 analyses, so random-effect models were selected (Table 4).

\section{BCG plus mitomycin $C$ vs. mitomycin $C$ alone}

Pooled data of 3 RCTs [35-37] didn't revealed BCG plus MMC significantly improved the RFS or PFS of patients with superficial $\mathrm{BCa}$ comparing with MMC alone (RFS: $\mathrm{HR}=0.854$, 95\% CI 0.663-1.099, $P=0.220$; PFS: HR=0.927, 95\% CI 0.482-1.784, $P=0.822$ ) (Figure 5A and B). Highrisk NMIBC were investigated in 2 studies $[35,37]$, integrated data also failed to detect remarkable advantage of combined therapy in reducing recurrence and progression rates comparing to MMC alone (RFS: $\mathrm{HR}=0.797,95 \%$ CI $0.477-1.330, P=0.385$; PFS: $\mathrm{HR}=0.829$, 95\% CI 0.387-1.773, $P=0.628$ ). There is no evidence of between-study heterogeneity in these analyses mentioned above (Table 4).

\section{BCG plus epirubicin vs. BCG alone}

Evidence from 3 studies [38-40] showed that combination of BCG and epirubicin could significantly prevent or delay the recurrence of superficial $\mathrm{BCa}(\mathrm{HR}=0.618 ; 95 \% \mathrm{CI} 0.384$ 0.993; $P=0.047$ ) (Figure 6A). The combination therapy had 
Table 4 Pooled results of recurrence-free survival, progression-free survival and publication bias of comparing various BCG treatment schedules and doses

\begin{tabular}{|c|c|c|c|c|c|c|c|}
\hline \multirow[t]{2}{*}{ Measurement } & \multirow[t]{2}{*}{$\mathrm{n}^{*}$} & \multirow{2}{*}{$\begin{array}{l}\text { Case or } \\
\text { case } \\
\text { control }\end{array}$} & \multicolumn{2}{|c|}{ Heterogeneity } & \multirow{2}{*}{$\begin{array}{l}\text { Pooled rate / HR } \\
\qquad(95 \% \mathrm{Cl})\end{array}$} & \multirow{2}{*}{$\begin{array}{l}\text { Begg's } \\
\text { test }(P)\end{array}$} & \multirow{2}{*}{$\begin{array}{l}\text { Egger's } \\
\text { test }(P)\end{array}$} \\
\hline & & & $P$ & $I^{2}(\%)$ & & & \\
\hline \multicolumn{8}{|l|}{ Recurrence-free survival } \\
\hline \multicolumn{8}{|l|}{ Superficial bladder cancer } \\
\hline Maintenance vs. Induction & 9 & $521 / 536$ & 0.150 & 12.0 & $0.516(0.425-0.627)$ & 0.602 & 0.353 \\
\hline Low-dose vs. Standard-dose & 7 & $998 / 1018$ & 0.831 & 0.00 & $1.162(1.051-1.285)$ & 1.000 & 0.126 \\
\hline BCG plus MMC vs. BCG & 6 & $416 / 563$ & $<0.001$ & 79.1 & $0.726(0.490-1.075)$ & 0.452 & 0.334 \\
\hline BCG plus MMC vs. MMC & 3 & $213 / 225$ & 0.297 & 17.7 & $0.854(0.663-1.099)$ & 0.296 & 0.132 \\
\hline BCG plus Epirubicin vs. BCG & 3 & $124 / 152$ & 0.652 & 0.00 & $0.618(0.384-0.993)$ & 1.000 & 0.538 \\
\hline BCG plus IFN-a2b vs. BCG & 2 & $383 / 342$ & 0.621 & 0.00 & $1.075(0.859-1.345)$ & 1.000 & NA \\
\hline \multicolumn{8}{|l|}{ High-risk NMIBC } \\
\hline Maintenance vs. Induction & 5 & $353 / 362$ & 0.272 & 22.4 & $0.515(0.411-0.646)$ & 0.086 & 0.032 \\
\hline BCG plus MMC vs. BCG & 3 & $314 / 298$ & $<0.001$ & 89.8 & $0.852(0.449-1.617)$ & 1.000 & 0.689 \\
\hline BCG plus MMC vs. MMC & 2 & $123 / 133$ & 0.119 & 58.7 & $0.797(0.477-1.330)$ & 1.000 & NA \\
\hline BCG plus Epirubicin vs. BCG & 2 & $100 / 102$ & 0.556 & 0.00 & $0.544(0.302-0.980)$ & 1.000 & NA \\
\hline \multicolumn{8}{|l|}{ Progression-free survival } \\
\hline \multicolumn{8}{|l|}{ Superficial bladder cancer } \\
\hline Maintenance vs. Induction & 7 & $452 / 488$ & 0.380 & 6.20 & $0.740(0.572-0.957)$ & 0.764 & 0.399 \\
\hline Low-dose vs. Standard-dose & 6 & $961 / 981$ & 0.829 & 0.00 & $1.151(0.853-1.554)$ & 0.452 & 0.112 \\
\hline BCG plus MMC vs. BCG & 3 & $314 / 298$ & 0.001 & 85.3 & $0.602(0.181-1.999)$ & 1.000 & 0.420 \\
\hline BCG plus MMC vs. MMC & 3 & $213 / 225$ & 0.827 & 0.00 & $0.927(0.482-1.784)$ & 1.000 & 0.493 \\
\hline BCG plus Epirubicin vs. BCG & 2 & $100 / 102$ & 0.980 & 0.00 & $0.513(0.132-1.987)$ & 1.000 & NA \\
\hline \multicolumn{8}{|l|}{ High-risk NMIBC } \\
\hline Maintenance vs. Induction & 5 & $353 / 362$ & 0.581 & 0.00 & $0.722(0.548-0.951)$ & 0.806 & 0.311 \\
\hline BCG plus MMC vs. BCG & 3 & $314 / 298$ & 0.001 & 85.3 & 0.602 (0.181-1.999) & 1.000 & 0.420 \\
\hline BCG plus MMC vs. MMC & 2 & $123 / 133$ & 0.813 & 0.00 & 0.829 (0.387-1.773) & 1.000 & NA \\
\hline BCG plus Epirubicin vs. BCG & 2 & $100 / 102$ & 0.980 & 0.00 & $0.513(0.132-1.987)$ & 1.000 & NA \\
\hline
\end{tabular}

NA not applicable. Significant datas are emphasized in bold.

* Number of included studies.

a more significant effect on preventing high-risk NMIBC from recurrence $(\mathrm{HR}=0.544 ; 95 \% \mathrm{CI} 0.302-0.980 ; P=0.043)$. Progression rate was reported in 2 studies including 202 high-risk NMIBC patients $[38,40]$, better outcome was observed in patients received combined therapy of BCG plus epirubicin, but this difference was not statistically significant $(\mathrm{HR}=0.513$; 95\% CI 0.132-1.987; $P=0.334$ ) (Figure 6B). The homogeneity among included studies was recognized by heterogeneity test (Table 4).

\section{BCG plus interferon $a-2 b$ vs. BCG alone}

Only RFS was discussed for this comparison, no significant difference was found between the two groups $(\mathrm{HR}=1.075 ; \quad 95 \% \quad \mathrm{CI} \quad 0.859-1.345 ; \quad P=0.527)$ (Figure 7), and no evidence of heterogeneity was presented (Table 4).

\section{Complications of treatment}

One of the most important indicators of complications was the percentage of patients who are unable to complete the treatment course because of side effects. Unfortunately, only therapy schedule of BCG plus MMC could be assessed by cessation rate due to side effects. As shown in Additional file 1: Figure S1, less cessation occurred in group of BCG plus MMC comparing to BCG or MMC alone, but no significant difference was detected $\quad(\mathrm{HR}=0.553,95 \%$ CI $0.170-1.797, \quad P=0.325$; $\mathrm{HR}=0.696$, 95\% CI 0.334-1.454, $P=0.335$; respectively).

Then the side effects accompanied with therapy were evaluated to oppose treatment efficacy. Numbers of limitation emerged hindering us from evaluating side effects caused by various therapy schedules and doses: 1) descriptors reported in individual articles were of great difference; 2) variety of terms were used to report 

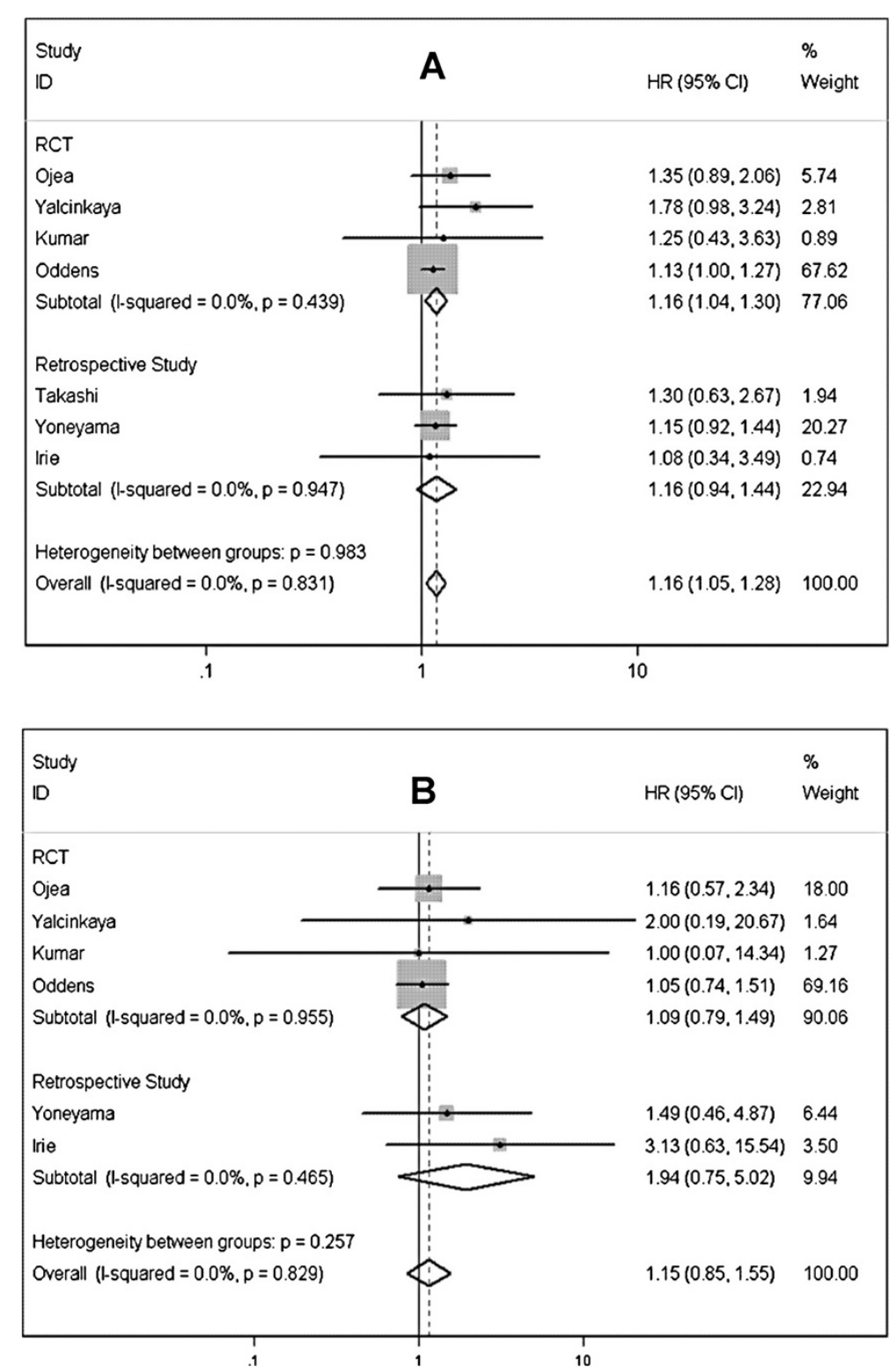

Figure 3 Forest plots of pooled results (A, recurrence-free survival; B, progression-free survival) for the low-dose group and standard-dose group.

complications; 3) only simple descriptions of complications were present in the most of included studies. The AUA guidelines panel for NMIBC combined complications into a set of categories [43]: bladder contracture, epididymitis/prostatitis/urethral infections, hematuria, lower urinary tract symptoms (LUTS) (including frequency, urgency, dysuria, etc.), fever/chills/flu symptoms, and systemic infection. According to the guideline panel, side-effects rates were present in a manner of 'maximal overlap', since it's unlikely that each patient had only one symptom. For example, a chilled man usually gets into fever. Maximal overlap indicates that different symptoms within the same category occurred simultaneously in the same patients [43].

Additional file 1: Table S1 listed common complications related to various treatments. The most common local and systemic side effects are LUTS and flu-like symptoms, respectively. Pooled risk differences of side effects that caused by different therapy schedules were showed in Additional file 1: Figure S2. The occurrence of local (e.g. hematuria and LUTS) and systemic (e.g. flu-like symptoms) side effects were both significantly elevated by maintenance therapy. Low-dose BCG only reduced the incidence of LUTS with a cautious 

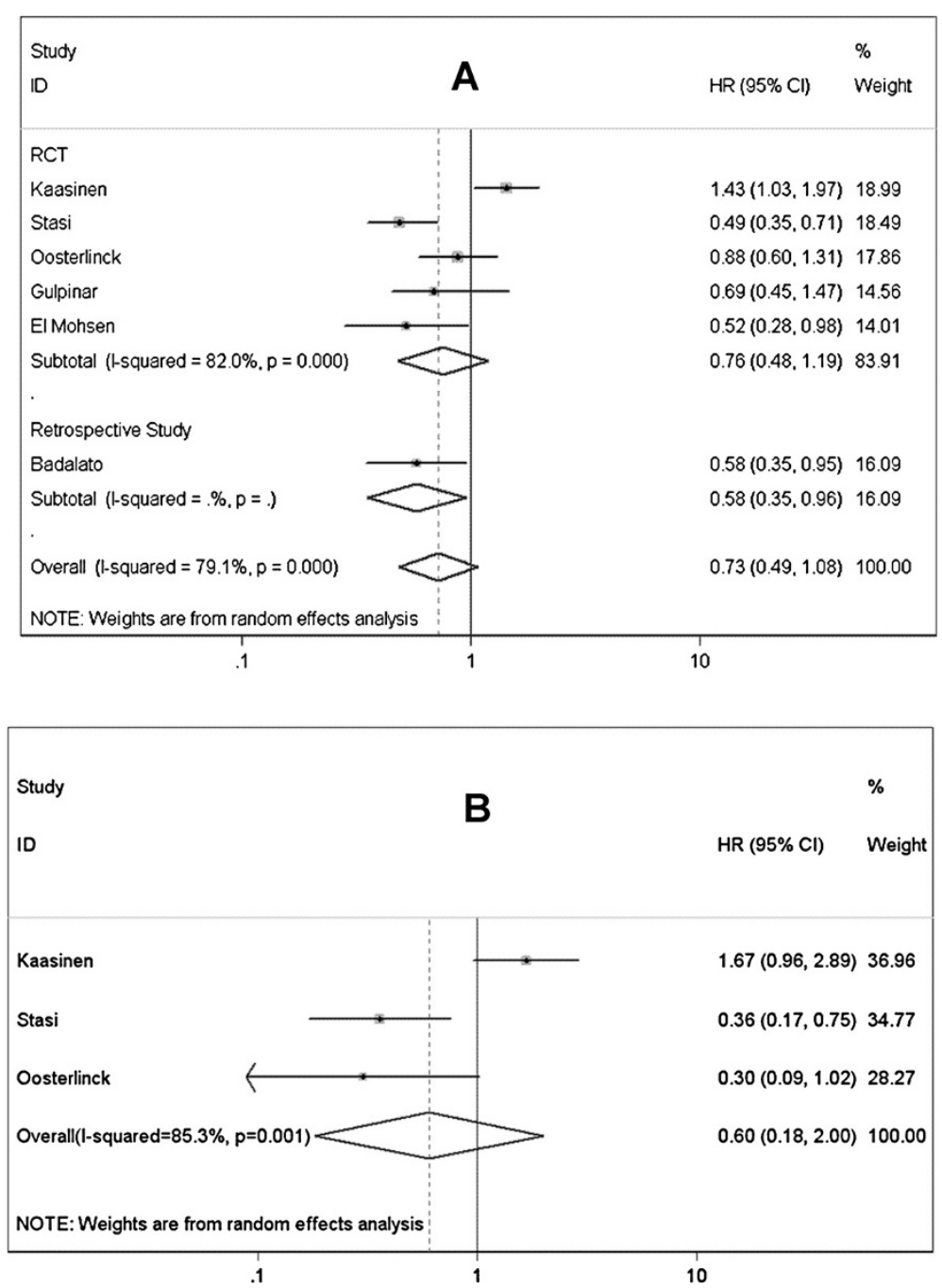

Figure 4 Forest plots of pooled results (A, recurrence-free survival; B, progression-free survival) for the BCG plus Mitomycin C group and BCG alone group.

significance, while no remarkable difference was present in other comparisons.

\section{Sensitivity analysis and publication bias}

For sensitivity analysis, we only included RCT. Subgroup data showed that BCG maintenance was no longer significantly prevent patients from getting the chance of progression (HR $=0.781 ; 95 \%$ CI $0.598-1.019 ; P=0.068$; Figure 2B). However, the obvious trend favoring BCG maintenance could be found still. And there was no significant difference between the results obtained from studies with the two distinct designs $(P=0.12)$. No other remarkable change was present in subgroup analyses stratified by study design. For difference between results obtained from trials and those with other study designs, the significance was only noted in Figure 1A $(P=0.03)$ Inclusion of the non-randomized studies might inap- propriately inflate the power of the analyses, thus the findings on RFS in this analysis should be interpreted with caution. Data from subgroup-analyses confirmed that non-RCT only affected strength rather than direction of the overall results. Another sensitivity analysis stratified by pathologic tumor stage was also conducted, as shown in Table 4, no variation was detected. The Begg's and Egger's tests (Table 4) revealed that significant publication bias existed in only 1 (BCG maintenance vs. induction for RFS in high-risk NMIBC group) of all comparisons performed in the present analysis.

\section{Discussion}

In this systematic review, we included 21 RCTs and 9 retrospective studies to evaluate the optimal schedule and dose of BCG for preventing recurrence and progression of superficial BCa. Pooled data revealed that BCG 

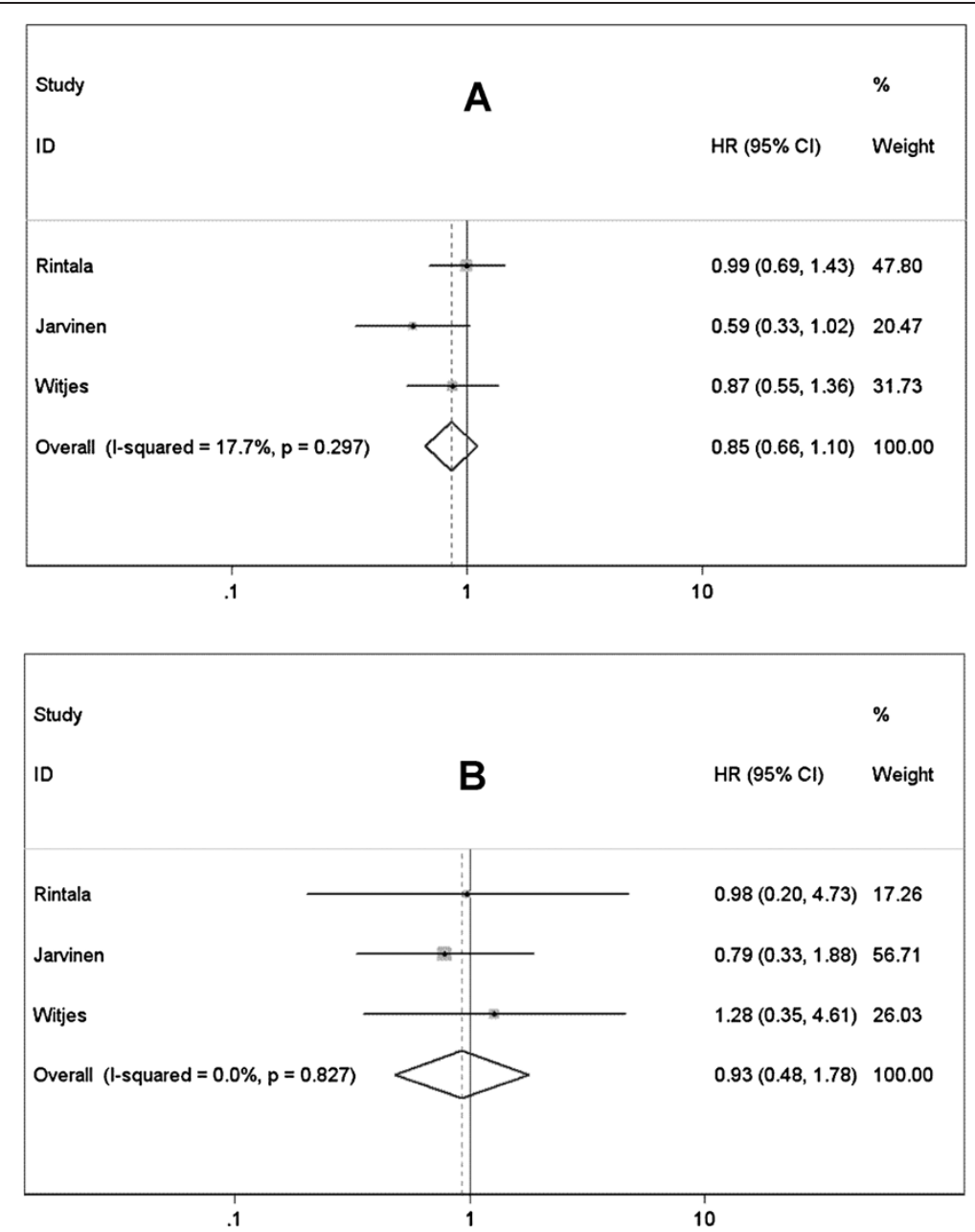

Figure 5 Forest plots of pooled results (A, recurrence-free survival; B, progression-free survival) for the BCG plus Mitomycin C group and Mitomycin $\mathrm{C}$ alone group.

maintenance could significantly improve RFS and PFS in patients with superficial $\mathrm{BCa}$, especially for patients with high-risk NMIBC. Our results were similar to the indirect evidence proved by Bohle et. al [3] and Sylvester et. al [4], which showed that maintenance therapy was associated with better outcomes, but sole induction course didn't seem to have superiority comparing with MMC. Accompanied with better clinical outcomes, incidence of adverse events was raised when patients receiving maintenance therapy. The potential benefits of BCG maintenance seemed to outweigh the risk of complications, even it was serious, and especially for patients with tumors that carried substantial risk of progression and might ultimate death from bladder cancer. In a high-quality cohort study, Decobert et al. [19] suggested that patients should be encouraged to tolerate at least 3 cycles of maintenance and to continue further instillations if well tolerated. However, serious side-effects caused by longterm BCG may be unworthy for those with low-risk lesions. Andius and Holmang showed that multiple instillation cycles may not be necessary for $\mathrm{pTa}$ and lowergrade tumors [20]. Unfortunately, there is no sufficient data to state if low-risk NMIBC could benefit from maintenance therapy. AUA guideline suggested that these risks and benefits should be discussed with the patients [43].

This meta-analysis shows that low-dose BCG may be defective in preventing tumor recurrence, which opposes to previous studies that clarified no significantly different RFS existed between low- and standard-doses BCG for patients with superficial $\mathrm{BCa}$, even for those with highrisk NMIBC (e.g. T1G3) [5,6,26]. In an effort to reduce the potential of complications caused by BCG (e.g. LUTS), pooled results have demonstrated sufficient efficacy using lower dose of BCG. Low-dose BCG can be chosen by patients with low-risk $\mathrm{BCa}$, and then adverse effects can be minimized. Nevertheless, there is no evidence support the hypothesis. 

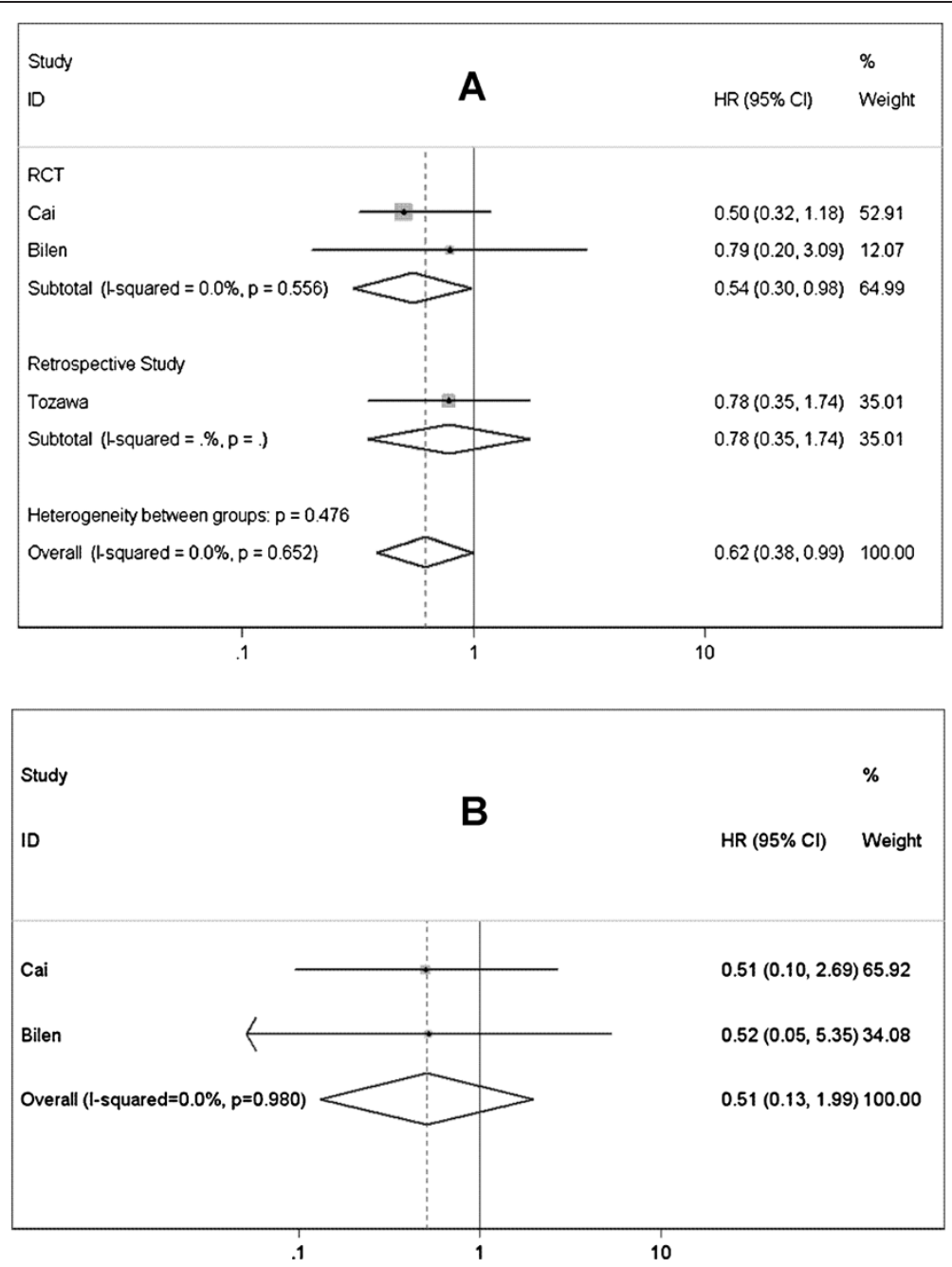

Figure 6 Forest plots of pooled results (A, recurrence-free survival; B, progression-free survival) for the BCG plus Epirubicin group and BCG alone group.

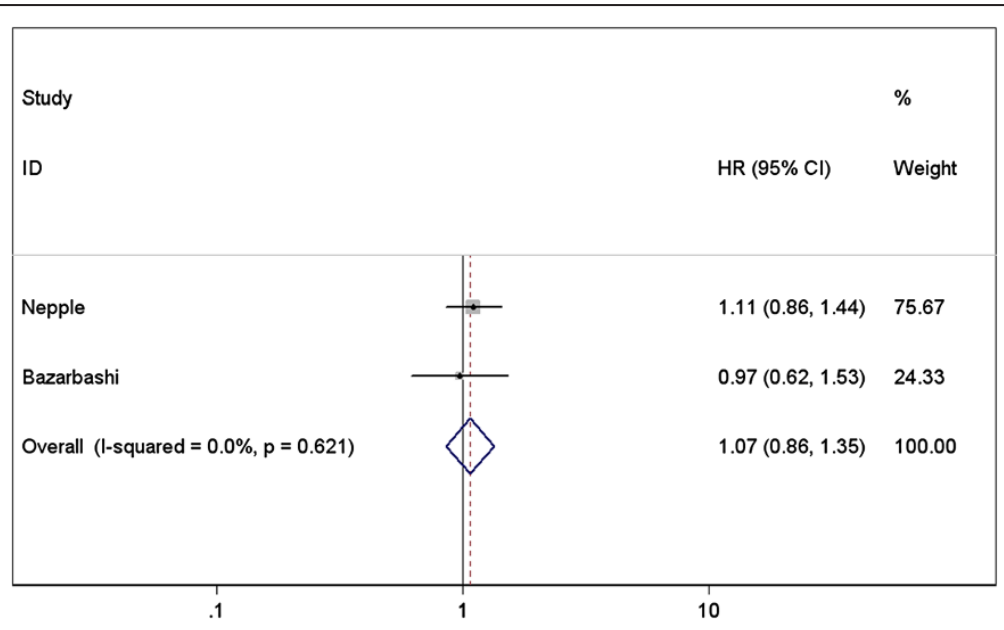

Figure 7 Forest plots of pooled results of recurrence-free survival for the BCG plus Interferon a-2b group and BCG alone group. 
Evidence in a cautious manner showed that sequential intravesical epirubicin could improve BCG efficacy regarding RFS in patients with superficial $\mathrm{BCa}$ (e.g. highrisk NMIBC). It was logical to deduce that treatment with sequential chemoimmunotherapy with two different antitumor mechanisms might be more active than monotherapy alone. However, pooled results of BCG combined with MMC diverged from the hypothesis because we did not find significant difference in terms of RFS and PFS between patients who received intravesical $M M C$ plus sequential BCG and those who received only BCG instillations. Significant heterogeneities were observed in this analysis and corresponding subgroupanalysis. Slight difference of treatment schemes existed in the therapy schedules and the different duration of follow-up period among the included studies may be responsible for them. Moreover, the combination use of BCG and MMC wasn't significantly superior to MMC alone. MMC may cause low efficacy of the combined treatment, because it can repress the immune response while preventing implantation of carcinoma. Basis researches were awaited to conduct the issue.

IFN- $\alpha 2 b$ is thought to increase the response of Th1 cell caused by BCG instillation through multiple approaches such as inhibiting interleukin-10 and enhance tumor necrosis factor-related apoptosis-inducing ligand release, ultimately lead to the suppression and subsequent destruction of urothelial carcinoma $[44,45]$. But we failed to observe a major efficacy after giving intravesical IFN- $\alpha 2 b$. However, IFN- $\alpha 2 b$ may be useful for patients with BCG refractory cases, for whom the BCG alone is insufficient to effectively awake the immune response to carcinoma. The final results of a national multicenter study of BCG plus IFN- $\alpha 2 b$ for treating superficial $\mathrm{BCa}$ confirmed the hypothesis [46]. The results showed a $59 \%$ and $45 \%$ recurrence-free rate of patient naive to BCG and those having BCG failure at a median follow-up of 24 months, respectively.

The overall results, except one, did not change remarkably after subgroup and sensitivity analyses. Publication bias was only observed in one subgroup analysis, which was proved by Egger's test. Our analysis combined the data from all studies that passed our predefined criteria; therefore, we are confident of the validity of our findings.

However, some inherent limitations of this meta-analysis should also be taken into account when interpreting our data. Firstly, most RCTs included in our analysis were of low-quality, which was caused by obvious side-effects which made it difficult to address double-blind. Another limitation of this study is the publication bias observed in subgroup analysis. This might impact the interpretation of the results because unpublished data may overturn this obtained result. So this result should be interpreted with caution. One reason for the bias may be that only English articles were searched, because other languages such as German were out of our ability. This selection might favor the positive studies that were more often published in English while the negative ones tended to be more often reported in native languages [47]. We attempted to minimize the publication bias by making our literature search as extensive as we could. Moreover, the clinical and pathologic stages of patients that were important to the oncologic prognosis were different in the included trials, which might substantially confound the presented results. Furthermore, slight difference of treatment schemes existed in the same therapy schedules, so the studies with standard schemes and enough follow-up time are expected. Finally, different duration of follow-up period among the included studies also affected the outcomes. To lessen the effect of follow-up period on synthetic results, HRs (time variable was taken into account) were prioritized in included studies.

\section{Conclusions}

Pooled result shows that BCG maintenance is associated with better oncologic outcomes (e.g. RFS and PFS). However, there is a higher incidence of adverse events. Low-dose BCG can't effectively prevent the recurrence of tumor, though a slightly reduced incidence of LUTS is observed. Compared with single BCG, combination with epirubicin may significantly reduce recurrence but not progression rate, and no more side-effect emerges. For both RFS and PFS, combination BCG with MMC or IFN- $\alpha 2 b$ is not superior to BCG and MMC alone. Given that the low-quality of the included studies can't be overcomed, large-volume, well-designed, RCTs with extensive follow-up are needed to confirm and update our findings.

\section{Additional file}

Additional file 1: Table S1. Side effects of various BCG therapy schedules and doses. Table S2. Quality assessment for RCTs using Jadad (5-ponit). Table S3. Quality assessment for retrospective study using Newcastle-Ottawa Scale. Figure S1. Summary of cessation-free survival for combination of BCG and Mitomycin C (A, BCG plus Mitomycin C vs. BCG alone; B, BCG plus Mitomycin C vs. Mitomycin C alone). Figure S2. Summary risk difference of side-effect for various BCG therapy schedules and doses.

\section{Abbreviations}

BCG: Bacillus calmette-guerin; BCa: Bladder cancer; NMIBC: Non-muscle-invasive bladder cancer; RFS: Recurrence-free survivals; PFS: Progression-free survival; MMC: Mitomycin C; Cl: Confidential intervals; IFN-a2b: Interferon a-2b; PRISMA: Preferred reporting items for systematic reviews and meta-analyses; HR: Hazard ratios; RR: Risk ratios; RD: Risk differences; LOE: Level of evidence; SC: Survival curve; RCT: Randomized-controlled trails; LUTS: Lower urinary tract symptoms.

Competing interests

All authors declared that they have no competing interest. 


\section{Authors' contributions}

YN originated the idea for the paper. YT and $\mathrm{HZ}$ carried out the systematic search. ZS, NJ and XN did the data extraction and analysis. SZ drafted the paper with assistance from $\mathrm{YN}$, YT and $\mathrm{KL}$. KL and SZ revised the manuscript according to the suggestions from the editor and reviewers. All authors reviewed the paper critically and have read and approved the manuscript for publication. All authors had full access to all the data in the study and take responsibility for the integrity of the data and the accuracy of the data analysis. YN are the guarantor. All authors have read and approved the final manuscript.

\section{Acknowledgements}

The work was supported by National Basic Research Program of China (Grant No. 2012CB518304), International S\&T Cooperation Program of China (ISTCP) (Grant No. 2012DFG32220) and Nature Science Foundation of Tianjin (Grant No. 11JCYBJC28400). Thank all of the authors of primary studies included in their meta-analyses.

\section{Author details}

'Department of Urology, Tianjin Institute of Urology, 2nd Hospital of Tianjin Medical University, Pingjiang Road 23, Tianjin, People's Republic of China. ${ }^{2}$ Department of Urology, Tianjin Third Central Hospital, Tianjin, People's Republic of China.

Received: 19 June 2013 Accepted: 20 June 2013

Published: 5 July 2013

\section{References}

1. Morales A, Eidinger D, Bruce AW: Intracavitary bacillus Calmette-Guerin in the treatment of superficial bladder tumors. J Urol 1976, 116(2):180-183.

2. Babjuk M, Oosterlinck W, Sylvester R, Kaasinen E, Bohle A, Palou-Redorta J, Roupret M: EAU guidelines on non-muscle-invasive urothelial carcinoma of the bladder, the 2011 update. Eur Urol 2011, 59(6):997-1008.

3. Bohle A, Bock PR: Intravesical bacille Calmette-Guerin versus mitomycin C in superficial bladder cancer: formal meta-analysis of comparative studies on tumor progression. Urology 2004, 63(4):682-686. 686-687.

4. Sylvester RJ, van der Meijden AP, Lamm DL: Intravesical bacillus CalmetteGuerin reduces the risk of progression in patients with superficial bladder cancer: a meta-analysis of the published results of randomized clinical trials. J Urol 2002, 168(5):1964-1970.

5. Yoneyama T, Ohyama C, Imai A, Ishimura H, Hagisawa S, Iwabuchi I, Mori K, Kamimura N, Koie T, Yamato T, et al: Low-dose instillation therapy with bacille Calmette-Guerin Tokyo 172 strain after transurethral resection: historical cohort study. Urology 2008, 71(6):1161-1165.

6. Kumar A, Dubey D, Bansal P, Mandhani A, Naik S: Urinary interleukin-8 predicts the response of standard and low dose intravesical bacillus Calmette-Guerin (modified Danish 1331 strain) for superficial bladder cancer. J Urol 2002, 168(5):2232-2235.

7. Yalcinkaya F, Kamis L, Ozteke O, Gunlusoy B, Yigitbasi O, Unal S: Prospective randomized comparison of intravesical BCG therapy with standard dose versus low doses in superficial bladder cancer. Int Urol Nephrol 1998, 30(1):41-44.

8. Gontero P, Bohle A, Malmstrom PU, O'Donnell MA, Oderda M, Sylvester R, Witjes F: The role of bacillus Calmette-Guerin in the treatment of nonmuscle-invasive bladder cancer. Eur Urol 2010, 57(3):410-429.

9. Moher D, Liberati A, Tetzlaff J, Altman DG: Preferred reporting items for systematic reviews and meta-analyses: the PRISMA statement. PLoS Med 2009, 6(7):e1000097.

10. Jadad AR, Moore RA, Carroll D, Jenkinson C, Reynolds DJ, Gavaghan DJ, McQuay HJ: Assessing the quality of reports of randomized clinical trials: is blinding necessary? Control Clin Trials 1996, 17(1):1-12

11. Wells G, Shea B, O'Connell D, Peterson J, Welch V, Losos M, Tugwell P: The Newcastle-Ottawa Scale (NOS) for assessing the quality of non-randomised studies in meta-analyses. Ottawa Hospital Research Institute Web site; 2012. [http://www.ohri.ca/programs/dinical_epidemiology/oxford.asp]. Accessed July 15, 2012.

12. Phillips B, Ball C, Sackett D, Badenoch D, Straus S, Haynes B, Dawes M: Levels of evidence and grades of recommendation, Oxford Centre for Evidencebased Medicine Levels of Evidence; 2012. [http://www.cebm.net/index.aspx? $\mathrm{o}=1025]$. Accessed July 15, 2012.
13. Parmar MK, Torri $V$, Stewart $L$ : Extracting summary statistics to perform meta-analyses of the published literature for survival endpoints. Stat Med 1998, 17(24):2815-2834.

14. Zhu S, Zhang H, Xie L, Chen J, Niu Y: Risk factors and prevention of inguinal hernia after radical prostatectomy: a systematic review and meta-analysis. J Urol 2013, 189(3):884-890.

15. Higgins JP, Thompson SG, Deeks JJ, Altman DG: Measuring inconsistency in meta-analyses. BMJ 2003, 327(7414):557-560.

16. Hinotsu S, Akaza H, Naito S, Ozono S, Sumiyoshi Y, Noguchi S, Yamaguchi A, Nagamori S, Terai A, Nasu Y, et al: Maintenance therapy with bacillus Calmette-Guerin Connaught strain clearly prolongs recurrence-free survival following transurethral resection of bladder tumour for non-muscle-invasive bladder cancer. BJU Int 2011, 108(2):187-195.

17. Okamura $\mathrm{T}$, Akita $\mathrm{H}$, Ando $\mathrm{R}$, Ikegami $\mathrm{Y}$, Naiki $\mathrm{T}$, Kawai $\mathrm{N}$, Tozawa $\mathrm{K}$, Kohri $\mathrm{K}$ : Single monthly bacillus Calmette-Guerin intravesical instillation is effective maintenance therapy to prevent recurrence in Japanese patients with non-muscle-invasive bladder cancer. Int I Clin Oncol 2011, 17(5):477-481

18. Koga H, Ozono S, Tsushima T, Tomita K, Horiguchi Y, Usami M, Hirao Y, Akaza H, Naito S: Maintenance intravesical bacillus Calmette-Guerin instillation for $\mathrm{Ta}, \mathrm{T} 1$ cancer and carcinoma in situ of the bladder: randomized controlled trial by the BCG Tokyo Strain Study Group. Int J Urol 2010, 17(9):759-766.

19. Decobert M, LaRue H, Harel F, Meyer F, Fradet Y, Lacombe L: Maintenance bacillus Calmette-Guerin in high-risk nonmuscle-invasive bladder cancer: how much is enough? Cancer 2008, 113(4):710-716.

20. Andius $\mathrm{P}$, Holmang S: Bacillus Calmette-Guerin therapy in stage $\mathrm{Ta} / \mathrm{T} 1$ bladder cancer: prognostic factors for time to recurrence and progression. BJU Int 2004, 93(7):980-984.

21. Palou J, Laguna P, Millan-Rodriguez F, Hall RR, Salvador-Bayarri J, VicenteRodriguez J: Control group and maintenance treatment with bacillus Calmette-Guerin for carcinoma in situ and/or high grade bladder tumors. J Urol 2001, 165(5):1488-1491.

22. Lamm DL, Blumenstein BA, Crissman JD, Montie JE, Gottesman JE, Lowe BA, Sarosdy MF, Bohl RD, Grossman HB, Beck TM, et al: Maintenance bacillus Calmette-Guerin immunotherapy for recurrent TA, T1 and carcinoma in situ transitional cell carcinoma of the bladder: a randomized Southwest Oncology Group Study. J Urol 2000, 163(4):1124-1129.

23. Badalament RA, Herr HW, Wong GY, Gnecco C, Pinsky CM, Whitmore WJ, Fair WR, Oettgen HF: A prospective randomized trial of maintenance versus nonmaintenance intravesical bacillus Calmette-Guerin therapy of superficial bladder cancer. J Clin Oncol 1987, 5(3):441-449.

24. Hudson MA, Ratliff TL, Gillen DP, Haaff EO, Dresner SM, Catalona WJ: Single course versus maintenance bacillus Calmette-Guerin therapy for superficial bladder tumors: a prospective, randomized trial. J Urol 1987, 138(2):295-298.

25. Ojea A, Nogueira JL, Solsona E, Flores N, Gomez JM, Molina JR, Chantada V Camacho JE, Pineiro LM, Rodriguez RH, et al: A multicentre, randomised prospective trial comparing three intravesical adjuvant therapies for intermediate-risk superficial bladder cancer: low-dose bacillus CalmetteGuerin $(27 \mathrm{mg}$ ) versus very low-dose bacillus Calmette-Guerin $(13.5 \mathrm{mg})$ versus mitomycin C. Eur Urol 2007, 52(5):1398-1406.

26. Irie A, Uchida T, Yamashita H, Matsumoto K, Satoh T, Koh H, Shimura S, Iwamura M, Baba S: Sufficient prophylactic efficacy with minor adverse effects by intravesical instillation of low-dose bacillus Calmette-Guerin for superficial bladder cancer recurrence. Int I Urol 2003, 10(4):183-189.

27. Takashi M, Wakai K, Ohno Y, Murase T, Miyake K: Evaluation of a low-dose intravesical bacillus Calmette-Guerin (Tokyo strain) therapy for superficial bladder cancer. Int Urol Nephrol 1995, 27(6):723-733.

28. Oddens J, Brausi M, Sylvester R, Bono A, van de Beek C, van Andel G, Gontero P. Hoelt W W, Turkeri L, Marreaud S, et al: Final results of an EORTCGU cancers group randomized study of maintenance bacillus CalmetteGuerin in intermediate- and high-risk $\mathrm{Ta}, \mathrm{T} 1$ papillary carcinoma of the urinary bladder: one-third dose versus full dose and 1 year versus 3 years of maintenance. Eur Urol 2013, 63(3):462-472.

29. Gulpinar O, Halilioglu OH, Gokce Ml, Gogus C, Baltaci S: The value of perioperative mitomycin $\mathrm{C}$ instillation in improving subsequent bacillus calmette-guerin instillation efficacy in intermediate and high-risk patients with non-muscle invasive bladder cancer: a prospective randomized study. Int Braz J Urol 2012, 38(4):474-479. 
30. Oosterlinck W, Kirkali Z, Sylvester R, Da SF, Busch C, Algaba F, Collette S, Bono A: Sequential intravesical chemoimmunotherapy with mitomycin $C$ and bacillus Calmette-Guerin and with bacillus Calmette-Guerin alone in patients with carcinoma in situ of the urinary bladder: results of an EORTC genito-urinary group randomized phase 2 trial (30993). Eur Urol 2011, 59(3):438-446.

31. Badalato GM, Hruby G, Razmjoo M, McKiernan JM: Maximizing intravesical therapy options: is there an advantage to the administration of perioperative mitomycin C prior to an induction course of BCG? Can J Urol 2011, 18(5):5890-5895.

32. El Mohsen M, Shelbaia A, EG S: Sequential chemoimmunotherapy using mitomycin followed by Bacillus Calmette-Guerin (MCC + BCG) versus single-agent immunotherapy (bcg) for recurrent superficial bladder tumors. Uro Today Int J 2010, 3(3) [http://www.urotoday.com/UIJ/Archive/ Volume-3/Issue-3-June-2010/sequential-chemoimmunotherapy-usingmitomycin-followed-by-bacillus-calmetteguerin-mcc-bcg-versus-singleagentimmunotherapy-bcg-for-recurrent-superficial-bladder.html]

33. Di Stasi SM, Giannantoni A, Giurioli A, Valenti M, Zampa G, Storti L, Attisani F, De Carolis A, Capelli G, Vespasiani G, et al: Sequential BCG and electromotive mitomycin versus BCG alone for high-risk superficial bladder cancer: a randomised controlled trial. Lancet Oncol 2006, 7(1):43-51.

34. Kaasinen E, Wijkstrom H, Malmstrom PU, Hellsten S, Duchek M, Mestad O, Rintala E: Alternating mitomycin C and BCG instillations versus BCG alone in treatment of carcinoma in situ of the urinary bladder: a nordic study. Eur Urol 2003, 43(6):637-645.

35. Jarvinen R, Kaasinen E, Rintala E, Group TF: Long-term results of maintenance treatment of mitomycin $\mathrm{C}$ or alternating mitomycin $\mathrm{C}$ and bacillus Calmette-Guerin instillation therapy of patients with carcinoma in situ of the bladder: a subgroup analysis of the prospective FinnBladder 2 study with a 17-year follow-up. Scand J Urol Nephrol 2012, 46(6):411-417

36. Witjes JA, Caris CT, Mungan NA, Debruyne FM, Witjes WP: Results of a randomized phase III trial of sequential intravesical therapy with mitomycin $C$ and bacillus Calmette-Guerin versus mitomycin $C$ alone in patients with superficial bladder cancer. J Urol 1998, 160(5):1668-1671. 1671-1672.

37. Rintala E, Jauhiainen $K$, Kaasinen E, Nurmi M, Alfthan O: Alternating mitomycin $C$ and bacillus Calmette-Guerin instillation prophylaxis for recurrent papillary (stages Ta to $\mathrm{T} 1$ ) superficial bladder cancer. Finnbladder Group. J Urol 1996, 156(1):56-59. 59-60.

38. Cai T, Nesi G, Tinacci G, Zini E, Mondaini N, Boddi V, Mazzoli S, Bartoletti R: Can early single dose instillation of epirubicin improve bacillus Calmette-Guerin efficacy in patients with nonmuscle invasive high risk bladder cancer? Results from a prospective, randomized, double-blind controlled study. J Urol 2008, 180(1):110-115.

39. Tozawa K, Okamura T, Sasaki S, Kawai N, Ito Y, Hayashi Y, Kohri K: Intravesical combined chemoimmunotherapy with epirubicin and bacillus Calmette-Guerin is not indicated for superficial bladder cancer. Urol Int 2001, 67(4):289-292.

40. Bilen CY, Ozen H, Aki FT, Aygun C, Ekici S, Kendi S: Clinical experience with BCG alone versus BCG plus epirubicin. Int J Urol 2000, 7(6):206-209.

41. Nepple KG, Lightfoot AJ, Rosevear HM, O'Donnell MA, Lamm DL: Bacillus Calmette-Guerin with or without interferon alpha-2b and megadose versus recommended daily allowance vitamins during induction and maintenance intravesical treatment of nonmuscle invasive bladder cancer. J Urol 2010, 184(5):1915-1919.

42. Bazarbashi S, Raja MA, El SA, Ezzat A, Ibrahim E, Kattan S, Kardar A, Peracha A, Lindstedt $E$, Hanash K: Prospective phase II trial of alternating intravesical Bacillus Calmette-Guerin (BCG) and interferon alpha IIB in the treatment and prevention of superficial transitional cell carcinoma of the urinary bladder: preliminary results. J Surg Oncol 2000, 74(3):181-184.

43. Hall MC, Chang SS, Dalbagni G, Pruthi RS, Seigne JD, Skinner EC, Wolf JJ, Schellhammer PF: Guideline for the management of nonmuscle invasive bladder cancer (stages Ta, T1, and Tis): 2007 update. J Urol 2007, 178(6):2314-2330.

44. Luo Y, Chen X, Downs TM, DeWolf WC, O'Donnell MA: IFN-alpha 2B enhances Th1 cytokine responses in bladder cancer patients receiving Mycobacterium bovis bacillus Calmette-Guerin immunotherapy. $\mathrm{J}$ Immunol 1999, 162(4):2399-2405.
45. Kayagaki N, Yamaguchi N, Nakayama M, Eto H, Okumura K, Yagita H: Type I interferons (IFNs) regulate tumor necrosis factor-related apoptosisinducing ligand (TRAIL) expression on human T cells: a novel mechanism for the antitumor effects of type I IFNs. J Exp Med 1999, 189(9):1451-1460.

46. Joudi FN, Smith BJ, O'Donnell MA: Final results from a national multicenter phase II trial of combination bacillus Calmette-Guerin plus interferon alpha-2B for reducing recurrence of superficial bladder cancer. Urol Oncol 2006, 24(4):344-348.

47. Egger M, Zellweger-Zahner T, Schneider M, Junker C, Lengeler C, Antes G: Language bias in randomised controlled trials published in English and German. Lancet 1997, 350(9074):326-329.

doi:10.1186/1471-2407-13-332

Cite this article as: Zhu et al:: Optimal schedule of bacillus calmetteguerin for non-muscle-invasive bladder cancer: a meta-analysis of comparative studies. BMC Cancer 2013 13:332.

\section{Submit your next manuscript to BioMed Central and take full advantage of:}

- Convenient online submission

- Thorough peer review

- No space constraints or color figure charges

- Immediate publication on acceptance

- Inclusion in PubMed, CAS, Scopus and Google Scholar

- Research which is freely available for redistribution 